\title{
La metamorfosis literaria de Publio Ovidio Nasón en la Novela del licenciado Vidriera
}

Este trabajo analiza la base mitológica que radica en el deambular inicial de Tomás Rodaja de la Novela del licenciado Vidriera. Durante este periplo el personaje se ve a sí mismo de vidrio. Se encuentra al principio de su camino con dos situaciones que recuerdan a los mitos de la Metamorfosis de Publio Ovidio Nasón: Deucalión y Pirra, y Níobe. Ambos comparten el elemento simbólico de la piedra en contraposición a la esencia vidriada del cuerpo de Tomás Rodaja. A su vez, esta oposición de materias propone un juego léxico que desenmascara al personaje de Ovidio en el propio licenciado Vidriera.

Palabras clave: Ovidio, Metamorfosis, mitología, vidrio, piedra

This study analyzes the mythological basis of the initial journey of Tomás Rodaja in Cervantes's Novela del licenciado Vidriera. During his journey, Rodaja thinks of himself as made of glass. Yet, at the beginning of his travels, he encounters two situations that recall the Ovidian myth of Deucalion and Pyrrha, and that of Niobe, both of which include the symbolic element of stone, as opposed to the glass essence of Rodaja's body. This opposition of elements is part of a lexical game whereby Cervantes unveils the person of Ovid in the licenciado Vidriera.

Keywords: Ovid, Metamorphoses, mythology, glass, stone

On this book, Reader, lay thy hand and swear, Ovid himself is Metamorphos'd here

-John Gower, Fasti (1640)

La historia literaria del Renacimiento ratifica la figura de Publio Ovidio Nasón como la más influyente de las letras clásicas. La autoridad que ejerce en este período deriva sobre todo de las numerosas reflexiones que residen en su obra. La especulación del poeta sobre la adaptabilidad de sus historias y la reencarnación de su persona cautivaron la imaginación de escritores 
que producen sus propias versiones ovidianas. ${ }^{\text {I }}$ En esta línea de imitadores sobresale la pluma de Ben Jonson, por ser el primero que resucita a Ovidio como personaje de carne y hueso en su pieza Poetaster (I60I). ${ }^{2}$ Igualmente, las letras hispanas del Siglo de Oro exhiben una gran fascinación por la obra y figura de Ovidio. Sin embargo, su persona como personaje imaginado está ausente de cualquier ficción literaria.

Este trabajo explica el mecanismo literario que Miguel de Cervantes emplea en la elaboración del proceso de transformación psicológica de Tomás Rodaja en vidrio, al igual que la razón letrada bajo la elección de esta sustancia vidriosa. Así pues, se propone una lectura de la Novela del licenciado Vidriera que saca a la luz la persona literaria de Ovidio encarnada y oculta en la de Tomás Rodaja, o como se le conoce después de su mutación en vidrio, el licenciado Vidriera. De esta manera, Cervantes despliega su habilidad como orfebre de las letras al crear un personaje de ficción que se identifica con la pluma de Ovidio. Para ello, se parte de dos eventos de la $N L V_{3}$ que sientan la base teórica con respecto al proceso de la transformación de la materia y sus agentes, los artesanos. Los hechos en la trama, el lanzamiento de piedras y el encuentro con una ropera salmantina, revelan una matriz ovidiana que razona la mutación mental del personaje principal, Tomás Rodaja, en vidrio. De esa manera, le sirven a Cervantes para fundar los andamios literarios e ideológicos en el proceso creacional de su ente vidriado. Estos sucesos ocurren tras la metamorfosis psicológica en vidrio del personaje principal, antes de que este se marche a la corte en Valladolid por mandato de un noble. Los dos acontecimientos revelan la presencia de dos mitos ovidianos, a saber, Deucalión y Pirra, y Níobe, que sirven para conectar a este nuevo personaje de vidrio con la figura poética de Ovidio. En esta línea, estos dos mitos camuflados tienen el componente de la piedra como denominador común de sus mutaciones. Ambos proponen una lectura de la transformación de Tomás Rodaja en vidrio dentro de un marco teorizador de corte ovidiano que apoya el empleo de esta sustancia.

La raíz exegética de estos dos hechos en la $N L V$ radica en la lectura antitética de sus mitos ovidianos correspondientes; es decir, el licenciado Vidriera experimenta unos acontecimientos que encierran elementos que apuntan en la dirección opuesta a lo que se cuenta en la versión original dentro de la Metamorfosis. ${ }^{4}$ La $N L V$ pone a prueba los conocimientos míticos de los lectores con esta adaptación antagónica que expone y argumenta el transformacional propósito de su novela; la metamorfosis máxima y por excelencia: la de Tomás Rodaja en un pseudo-Ovidio, un personaje que en la cacofonía de su estructura corpórea rememora al vate por excelencia: vidrio-Ovidio.5 El licenciado Vidriera participa ahora en sucesos míticos de 
la Metamorfosis que se adaptan a la realidad histórica del momento. Simula a la persona de Ovidio en su composición vidriosa gracias a una lectura distorsionada de su nombre. Por un lado, el licenciado Vidriera se introduce como un doppelgänger literario dentro de episodios míticos que su padre poético, Ovidio, ya formuló en la Metamorfosis. Por otro, Cervantes emula literariamente al creador de la Metamorfosis. Se presenta como artesano letrado que crea con genio y sutileza a un personaje que se asemeja a Ovidio gracias al parecido que su psique vidriosa mantiene con el nombre del poeta. De esta manera se erige como transformador literario sin par cuando metamorfosea la humanidad de Tomás Rodaja en una corporeidad que imita y recuerda al genio letrado de las metamorfosis poéticas. ${ }^{6}$

La crítica cervantina muestra una inclinación especial por la transformación imaginaria en vidrio que padece el personaje principal de Tomás Rodaja de la $N L V$ dentro de las Novelas ejemplares. Su locura abre el camino a estudios que tratan de la esquizofrenia (Vallejo; Górriz); su paradigma bipolar, y la libertad y alienación (Joset, "Bipolarizaciones textuales" y "Libertad y enajenación"); y la relación entre individuo y sociedad (Encinar).7 Esta transición a un cuerpo vidrioso impulsó a investigadores a buscar individuos de carne y hueso, o de ficción que pudieran haber inspirado a Cervantes (Hainsworth; González de Amezúa; Segre). La triple onomástica del personaje principal: Tomás Rodaja, licenciado Vidriera y licenciado Rueda llama la atención de Francisco García Lorca, quien conecta esta triple presencia con las tres etapas básicas de la novela. El crítico sugiere la denominación a secas de Tomás por diecisiete veces como un problema de personalidad (García Lorca 163). En esta línea, María Antonia Garcés estudia la fracturación de su patronímico y psicosis con un acercamiento lacaniano (225). Con todo, la inestabilidad de su onomástica y persona contribuyen a formular un personaje camaleónico que se oculta tras esta lucha con su ipseidad. Al mismo tiempo, la decisión de Tomás Rodaja de vestirse de papagayo para emprender un viaje por Italia en compañía del capitán don Diego de Valdivia encierra una lectura más allá de su mera colorida vestimenta. Este traje lleno de tonalidades apunta a un engranaje de referencias literarias entre la poesía elegíaca de Ovidio y Catulo. Ovidio adopta la figura del papagayo para aludir indirectamente al gorrión elegíaco de la obra de Catulo. Esta imagen alada contiene una exégesis referencial de corte poético, y le sirve a Tomás Rodaja para adquirir una identidad ovidiana (Hidalgo, "El ethos elegíaco" 102). Después de este travestismo aviar que apunta a Ovidio, sobresale el paso de la materia humana a la de vidrio, es decir, el momento en el que Tomás Rodaja enloquece e imagina que su cuerpo está formado de vidrio. Este estado psicológico de Tomás Rodaja invita a escudriñar en su materia 
corpórea una valoración en línea con otro estrato ovidiano. La imaginada materialidad vidriada del cuerpo de Tomás Rodaja encierra un juego referencial al padre literario por excelencia de las metamorfosis literarias: Publio Ovidio Nasón. ${ }^{8}$ De hecho, la presencia de Ovidio se manifiesta directamente en la $N L V$, y en boca del licenciado Vidriera, cuando este marcha a Valladolid por orden de un noble. Allí, un estudiante le pregunta si él es poeta, pues su ingenio así lo demuestra: "- Hasta ahora no he sido tan necio, ni tan venturoso. - No entiendo eso de necio y venturoso - dijo el estudiante. Y respondió Vidriera: - No he sido tan necio que diese en poeta malo, ni tan venturoso que haya merecido serlo bueno" (Cervantes, Novelas 57-8). 9

El primer suceso tras la mutación psíquica de Tomás Rodaja en vidrio encierra toda una conexión intrínseca con la Metamorfosis de Ovidio. Una multitud de chicos le arroja trapos y piedras. La litología letrada del elemento "piedra" prima a lo largo de toda la obra ovidiana, y es la base y resultado de un gran número de metamorfosis. De la misma manera que Ovidio transforma sus deidades y personajes en esta materia rocosa, Cervantes contrapone, con la metamorfosis estelar de Tomás Rodaja, la maleabilidad y transparencia del vidrio a la resistencia y opacidad de este mineral. A su vez, expresa con esta singular mutación su contraposición a las que ocurren en la Metamorfosis, por lo que disputa con Ovidio mediante el empleo de una sustancia que se opone al símbolo distintivo del poema épico. Así, Cervantes conoce este tropo letrado de la Metamorfosis y lo subvierte ahora para emplearlo en la raíz transformacional de la $N L V$. Ovidio y Cervantes son artífices de la materia física, y transformadores poéticos de la tradición literaria. En esta línea, Ovidio despliega un variado abanico de mutaciones en su obra maestra, la Metamorfosis, mientras que Cervantes desfigura y distorsiona a Tomás Rodaja en un personaje de vidrio que imita en la materia vidriosa de su ser al padre letrado y excelso de la Metamorfosis. Ambos elementos, piedra y vidrio, son los exponentes que identifican las excelsas habilidades literarias de sus maestros, Ovidio y Cervantes. Ahora, este segundo compite mediante la mutación más inesperada, la de un personaje de ficción que piensa que posee una naturaleza vidriada, y cuya sustancia es contraria a la metáfora maestra de la Metamorfosis, vidrio contra piedra. Cervantes imita y reduce a su modelo poético, Ovidio, con esta fina y sugestiva transfiguración, el vidrio. Así pues, despliega su talento para transformar, y dialoga con el genio de la Metamorfosis al enfrascarlo en este elemento vidriado. No hay cambio más eminente que la propia mutación del creador de las mutaciones míticas en la transformación de una sustancia que distorsiona su onomástica y se contrapone al tropo maestro de su obra sublime. 
La $N L V$ cuenta la llegada de una mujer que se enamora de Tomás Rodaja después de su periplo por tierras italianas y flamencas. Ésta, ante las negativas de Tomás Rodaja que sentía una mayor inclinación por sus libros, opta por envenenarle con un membrillo toledano con la esperanza de doblegar su voluntad. Los efectos del veneno envían a Tomás Rodaja a pasar seis meses de convalecencia. Durante este tiempo se cura de la enfermedad física que padecía, pero su estado mental queda dañado. A partir de aquí, el texto enfatiza su condición demente al creer que su cuerpo está hecho todo de vidrio: "porque quedó sano, y loco de la más extraña locura que entre las locuras hasta entonces se había visto. Imaginóse el desdichado que era todo hecho de vidrio" (Cervantes, Novelas 53). ${ }^{\circ}$ Sus amigos intentan convencerle sin éxito de que abandone la creencia irracional de que su ser posee una cualidad vidriosa. Tomás Rodaja pide a sus amigos que le dejen andar libre por la ciudad tras tenerle encerrado por varios días. A partir de aquí suceden dos situaciones que conectan con la Metamorfosis de Ovidio. La primera de ellas narra el encuentro de un grupo de muchachos que le rodean. Este les pide que mantengan la distancia a la hora de hablarle debido a su frágil condición. Los jóvenes le desobedecen, y comienzan a arrojarle trapos y piedras, con la suerte de que ninguno de estos lanzamientos le alcanza. Unos hombres salen en su ayuda, y consiguen alejar a estos muchachos que después deciden acompañarle y oírle, en lugar de continuar con sus tiros. Lo destacado de este encuentro es su colocación inicial justo después de que Tomás Rodaja piensa que su cuerpo se ha transformado en vidrio y, sobre todo el hecho de que estos le lanzan piedras. Este incidente cruel pasa como una travesura más de un grupo de chicos que amenazan con desintegrar la corporeidad vítrea del personaje principal. Sin embargo, su emplazamiento primerizo, justo tras imaginarse que posee un cuerpo de vidrio, y el lanzamiento de materias rocosas, recuerdan uno de los mitos iniciales del libro primero de la Metamorfosis: Deucalión y Pirra. ${ }^{I I}$

La piedra, en todas sus variantes y representaciones, es la imagen dominante en la Metamorfosis. Ocupa el primer lugar por encima del tema del amor y el de las artes plásticas, y por ello su presencia deliberada en el libro primero:

Of all the themes in the Metamorphoses, none recurs either as frequently or as patently as that of the stone. ... Because the stone image has such an important function in the scheme not only of the whole work but also of each individual book, Ovid has at the outset equipped us with the Deucalion and Pyrrha episode (I.313-425), a complete catalogue of the vocabulary to be used throughout and a forecast of the countless permutations the image will undergo. (Bauer 2) ${ }^{\mathrm{r} 2}$ 
El símbolo de la piedra aparece en ambas historias con una dualidad enfrentada. Tiene un valor destructivo en su contacto con el vidrio en la $N L V$, mientras que posee un valor regenerador al tocar la tierra en el mito de Deucalión y Pirra. Este último forma parte del libro primero de la Metamorfosis y viene tras el suceso del gran diluvio que acaba con la humanidad (I.315-415).13 La habilidad artesanal de ambos personajes, Deucalión y Pirra, como escultores de la vida humana viene marcada en los versos anteriores por una divinidad que se encarga de moldear en primer lugar la unidad material que forma la naturaleza: Caos. Luego, se le considera a este dios el progenitor del ser humano, pero pronto se le relega, y la figura de Prometeo adquiere la supremacía fundadora del ser de carne y hueso. El texto describe a ambos, Deucalión y Pirra, con características que pertenecen al mundo de las artes plásticas. La divinidad posee la peculiaridad de un individuo que emplea sus habilidades manuales para la confección de una obra: "His quoque non passim mundi fabricator habendum / aëra permisit" "Tampoco a los vientos concedió el artífice del mundo el libre uso del aire"; I.57-58; Ruiz 8). La palabra "fabricator" y su familia léxica comprenden este universo creacional de las manualidades al que pertenece la deidad. ${ }^{14}$ Después, Prometeo toma el papel de iniciador de la raza humana según la tradición mítica. ${ }^{15}$ La destrucción de la raza humana por orden de Júpiter con la llegada del diluvio introduce a los personajes de Deucalión y Pirra en la historia de este libro primero de la Metamorfosis. Deucalión lamenta no tener las habilidades artesanas de su padre, Prometeo, a la hora de repoblar la tierra, y su lamentación recuerda su ascendencia artística: "O utinam possim populos reparare paternis / artibus atque animas formatae infundere terrae!” “"iOjalá pudiera yo restablecer la población del mundo con las facultades de mi padre y derramar vida en la tierra después de modelarla!"; I.363-64; Ruiz 21-22). ${ }^{16}$ Ambos visitan el oráculo de la divinidad Temis en busca de una solución a la desolación que les rodea. Reciben una respuesta que les sobrecoge por lo inusual de su designio, y tras evaluarla y descifrar el mensaje que trae oculto, aceptan la voluntad divina y comienzan a ejecutarla: "Discedunt uelantque caput tunicasque recingunt / et iussos lapides sua post uestigia mittunt ("Se alejan, cubren sus cabezas, se desciñen las túnicas, y van tirando sobre sus huellas las piedras prescritas"; I.398-99; Ruiz 23). A continuación, se detalla la mutación que estas piedras padecen, y el surgimiento de la esencia mortal:

Saxa (quis hoc credat, nisi sit pro teste uetustas?) / ponere duritiem coepere suumque rigorem / mollirique mora mollitaque ducere formam. / Mox ubi creuerunt 
naturaque mitior illis / contigit, ut quaedam, sic non manifesta uideri / forma potest hominis, sed, uti de marmore coepta, / non exacta satis rudibusque simillima signis.

Los pedruscos (¿quién lo creería si no lo atestiguara la antigua tradición?) empezaron a despojarse de su dureza y de su rigidez, a ablandarse conforme pasaba el tiempo, y, una vez ablandados, a tomar forma. Después, cuando crecieron y adquirieron una naturaleza más suave, podía ya parecer aquello un algo de figura humana, aunque todavía no resultaba evidente, sino que era como una obra empezada en mármol, no bien terminada aún y semejante a las estatuas a medio hacer. (I.400-406; Ruiz 23)

Este proceso de variación de materia rocosa a humana interesa por la conexión que guarda con el mundo artístico, y en concreto, la escultura: "The process defines both art and life, and it joins them. The evolution that Ovid describes is strikingly parallel to artistic creation, for this is a Promethean form of sculpture that seems to arise out of the matter itself" (Barkan 643).17 Así, la evolución de estos peñascos humanoides semeja el procedimiento de creación que experimenta una masa física bajo las habilidades de un maestro artesano y, para el caso, un escultor. Deucalión y Pirra poseen las dotes creativas de los cinceladores, e igualmente recuerdan la importancia de los sentidos de la visión y el tacto, que son los más destacados de estos artistas.

La lluvia de piedras que Tomás Rodaja aguanta cuando se imagina que adquiere una complexión de vidrio rememora, aunque de modo invertido, el mito de la creación de los humanos a cargo de Deucalión y Pirra. En tal mito, las piedras son entidades cargadas de vida que, después de ser lanzadas y entrar en contacto con la tierra, se regeneran en materia humana, y todo ello guarda semejanza con un proyecto artístico, la escultura. Los pedruscos míticos de Deucalión y Pirra poseen habilidades regeneradoras mientras que los de la $N L V$ causan daño físico. Por su parte, la $N L V$ presenta una transformación en la que un ser animado, Tomás Rodaja, sucumbe, tras tomar un veneno, para transformarse en una imaginada forma material e inhumana, el vidrio. La $N L V$ inserta la invención de su personaje ficticio, el licenciado Vidriera, en línea con este mito de creación de la Metamorfosis. De esta manera, la materia corpórea de vidrio en la $N L V$ contradice la semilla fundacional, la piedra, del mito de Deucalión y Pirra de la Metamorfosis. Ambas obras, la Metamorfosis y la $N L V$, comparten una mutación que sirve de futuras transformaciones que tienen como estado de cambio la piedra, y en el primer caso, como origen de la raza humana. En el caso de la novela, la conversión corpórea en vidrio es la base estructural del texto. Mientras que Ovidio utiliza esta materia inerte, piedra, como el tropo maestro de otras 
historias transformacionales a lo largo de la Metamorfosis, en la $N L V$ se transforma a un ser de carne y hueso, Tomás Rodaja, en vidrio. Es decir, la $N L V$ contradice la metáfora maestra y ovidiana de la piedra con la materia y estado del personaje en vidrio. Así pues, la $N L V$ no es un cúmulo de historias que tienen como denominador común una conversión de individuos y seres en otra materia común, piedra. Con todo, el lanzamiento de piedras inaugura el nacimiento de la metamorfosis fundacional de la novela, la de Tomás Rodaja en vidrio.

El personaje de Pirra aparece durante todo este proceso como una exégeta que interpreta literalmente los dictados del oráculo: "Pyrrha shows herself a reader of the letter, accepting language at its surface value. Only Deucalion is able to uncover the hidden meaning of the oracular text, realizing that their mother is the earth and her bones are stones. ... Deucalion, on the other hand, reads not in the surface manner of Narcissus but in such a way as to decode and realize the oracular metaphor" (Huot 869). La adivinanza del oráculo que confunde en un principio a Pirra anima a buscar una interpretación alegórica para su desciframiento, por lo que este acertijo juega un papel interpretativo que el lector debe afrontar en la $N L V$. Con ello, su mensaje incita a escudriñar en la letra impresa y no confiar en una lectura literal $a$ priori, sino buscar una exégesis figurativa para estos sucesos ordinarios que componen y rodean el positivismo de la trama de la $N L V$. La leyenda de Deucalión y Pirra invita a no tomar al pie de la letra los acontecimientos que se narran, y exige una interpretación que se desvíe de la literalidad del texto. Por lo tanto, el acontecimiento inicial del lanzamiento de piedras encierra una exégesis que expone el trasfondo artístico de su colocación y funcionamiento dentro de la trama y se conecta también con futuros eventos. La piedra en el mito de Deucalión y Pirra tiene poderes regeneradores tras su lanzamiento, mientras que en la $N L V$ adquiere una cualidad destructora y dañina, pues busca herir la personalidad vidriosa de Tomás Rodaja. Esta oposición de valores se presenta a su vez como principio instructivo para descifrar realidades futuras, ya que el efecto negativo que la piedra adquiere en la $N L V$ se contrapone a su intención positiva en la historia de Deucalión y Pirra. De esta manera, la Metamorfosis es la base literaria de donde parten historias que luego se invierten en la $N L V$.

De vuelta a la $N L V$, se cuenta que una multitud siempre sigue a Tomás Rodaja para escuchar sus dichos tras su cambio psicológico en materia de vidrio. Como parte de su peregrinaje, se narra el encuentro que tiene con una ropera de Salamanca. Este suceso cobra gran relevancia en conexión con el anterior altercado en el que los chicos le lanzan piedras pues, al igual que el lanzamiento de las piedras sugiere el mito de Deucalión y Pirra, la incapacidad para llorar de la ropera junto con el dicho del ahora licenciado 
Vidriera recuerdan el mito de Níobe. Este personaje mitológico padeció, debido a su orgullo, la ira de los hijos de Latona, Apolo y Diana, quienes fulminaron a su prole de catorce vástagos. Esta desgracia le produjo tanto pesar que nunca dejó de llorar, y en su gemir infinito acabó por transformarse en piedra. Así pues, la ropera se lamenta del estado de vidrio de Tomás Rodaja, a partir de ahora, licenciado Vidriera, y éste le responde con una cita bíblica que el marido toma a despecho: "- En mi ánima, señor Licenciado, que me pesa de su desgracia; pero ¿qué haré, que no puedo llorar? Él se volvió a ella, y muy mesurado le dijo: - Filiae Hierusalem, plorate super vos et super filios vestros. Entendió el marido de la ropera la malicia del dicho y díjole: - Hermano licenciado Vidriera - que así decía él que se llamaba -, más tenéis de bellaco que de loco. - No se me da un ardite respondió él -, como no tenga nada de necio" (Cervantes, Novelas 55).

La insuficiencia lacrimosa de la ropera y el mensaje de la sentencia bíblica, que menciona a su prole, posibilitan pensar en una historia mítica en la que, de manera contraria, el personaje principal llora desconsoladamente la muerte de sus hijos, Níobe. El dueto expuesto anteriormente, Deucalión y Pirra, y el lanzamiento de piedras en la $N L V$, contraponen los efectos de su materia principal, la piedra. En el primer caso tiene efectos regenerativos, y en el segundo destructores. Igualmente, el mito de Níobe recoge ahora esta contraposición. Se presenta a una mujer que padece de una incapacidad ocular y es madre de varios vástagos. En esencia, la supuesta xeroftalmía de la ropera contradice el incesante lloro de Níobe al final de su mito. A su vez, ocurre con esta narración mítica de Níobe un cambio que contradice el mito de Deucalión y Pirra, pero que complementa el estado vítreo del licenciado Vidriera. En el primer caso, Deucalión y Pirra, se aprecia el paso de materia inerte a vida, mientras que ahora se ve la permuta de dos seres animados a inanimados: Tomás Rodaja en vidrio, y Níobe en piedra. Lo interesante de este mito también es el estado rocoso que ella padece, y que sigue así la línea temática del suceso anterior de los jóvenes lanzadores de piedra y también del mito de Deucalión y Pirra. Consecuentemente, se crea un nexo pétreo entre ambas situaciones.

Jesús exhorta a las mujeres de Jerusalén a que se lamenten de su propia situación y la de sus hijos con esta cita que pertenece al evangelio según san Lucas: "Lo seguía mucha gente del pueblo y mujeres, que se daban golpes de pecho y se lamentaban por él. Jesús se volvió a ellas y les dijo: 'Hijas de Jerusalén, no lloréis por mí; llorad por vosotras y vuestros hijos, porque vienen días en los que se dirá: Dichosas las estériles, los vientres que no han dado a luz y los pechos que no han amamantado"' (Santa Biblia, Lucas 23.2730; 15I2). La fertilidad de estas mujeres que lamentarán el funesto desenlace de sus vástagos mediante un incontrolable e incesante llanto resuena en la 
memoria del licenciado Vidriera cuando esta salmantina ropera le comenta su incapacidad de llorar. Fuera de este marco bíblico, la mitología clásica cuenta la historia de una madre que lamentó con lágrimas infinitas la muerte de su prole, el arquetipo de la madre desconsolada, Níobe. La versión completa del mito se encuentra en el libro sexto de la Metamorfosis (VI.I46-3I2; Ruiz 23-30). Aquí, Níobe se jacta de haber engendrado más hijos que Leto, la madre de Apolo y Diana, llegando a un total de catorce, siete varones y siete hembras. Leto refiere la afrenta a sus hijos, y ambos sin tardanza ejecutan con sus flechas a la descendencia varonil de Níobe y, acto seguido, a su prole femenina. Níobe entra en un estado de conmoción con la fulminación inesperada de sus catorce vástagos. La historia concluye con su transformación en una piedra que no deja de echar agua y recuerda a una estatua:

Orba resedit / exanimes inter natos natasque uirumque / deriguitque malis: nullos mouet aura capillos, / in uultu color est sine sanguine, lumina maestis / stant inmota genis: nihil est in imagine uiuum. / Ipsa quoque interius cum duro lingua palato / congelat, et uenae desistunt posse moueri; / nec flecti ceruix nec bracchia reddere motus / nec pes ire potest; intra quoque uiscera saxum est. / Flet tamen et ualidi circumdata turbine uenti / in patriam rapta est; ibi fixa cacumine montis / liquitur, et lacrimis etiam nunc marmora manant.

Sin prole ya se sentó entre sus hijos, hijas y marido sin vida, y por su desgracia se tornó rígida: la brisa no mueve sus cabellos, en su cara hay un color exangüe, sus ojos están inmóviles en sus sombrías mejillas, y nada hay vivo en aquella figura. También por dentro la misma lengua se le hiela adherida al paladar duro, y las venas cesan de tener movimiento; ni el cuello puede doblarse ni los brazos hacer movimientos ni el pie caminar; incluso en el interior de las vísceras hay piedra. Llora sin embargo, y rodeada por un torbellino de poderoso viento fue trasladada a su patria; sujeta allí en la cumbre de la montaña, se licúa, y todavía ahora siguen los mármoles manando lágrimas. (VI.3OI-I2; Ruiz 29-30)

El breve intercambio de palabras que el licenciado Vidriera mantiene con la ropera y el marido posibilita un escenario literario más allá del bíblico. Entra en escena la historia mítica de Níobe que llora desconsolada y para siempre la muerte de su progenie. Esta tragedia horrenda hace que la víctima padezca la transformación inversa que el mito de Deucalión y Pirra narra. Es decir, ahora la materia animada se convierte en sustancia inerte. Níobe yace petrificada y con lágrimas incesantes a manera de estatua. En este sentido, los dos mitos ejemplifican dos posibles cambios de sustancia. Se pasa de un estado rocoso y sin vida a una fase de existencia humana, y 
viceversa. Estas dos historias ovidianas exponen la habilidad artística de su autor, Ovidio, para transformar entidades en ambas direcciones de cambio. En este sentido, la $N L V$ coloca un nivel más de cambio, de manera que además de ofrecer las dos posibles mutaciones con ambos mitos ovidianos (de piedra a seres humanos, y de ser humano a piedra) se brinda una nueva posibilidad, la de metamorfosear un ser humano en vidrio, o todavía más, un sujeto ficticio que imita una figura literaria: Tomás Rodaja en vidrio, luego de vidrio a O-vidrio/Ovidio; y finalmente, en el licenciado Vidriera. Se consigue así un trío de mutaciones, y es esta última, en vidrio, la que sobresale porque logra una variación que encierra en su estado vítreo al padre por excelencia de las metamorfosis literarias, Ovidio.

La mutación axial de la $N L V$ es la de Tomás Rodaja en vidrio, y a partir de esta, el personaje adquiere el sobrenombre de licenciado Vidriera. Esta vitrificación le confiere una nueva identidad física. También, éste despliega a partir de ahora una sorprendente erudición que deriva del modelo literario al que imita, Ovidio. De hecho, le preguntan si era poeta cuando se marchó a Valladolid debido al ingenio que mostraba. La NLV inserta, después de este cambio psicológico, dos situaciones que pasan de ser meras escenas a ser dos realidades que reflexionan sobre el acto de la transformación, a saber, el ataque de piedras por niños y la conversación con una ropera y su esposo. Una y otra parten de los mitos de Deucalión y Pirra, y Níobe de la Metamorfosis de Ovidio. En estos, la piedra es la sustancia de la que surge el cambio y a donde concluye. Esta sustancia rocosa, que es la materia y metáfora distintiva que predomina en la Metamorfosis, sucumbe ante el elemento del vidrio en la $N L V$. La $N L V$ mantiene la mutación inusual de un ser en materia de vidrio como fuerza motriz de su trama, de manera que se opone al componente de la piedra. Ambos mitos ovidianos están conectados por este constituyente, la piedra, y lo que es más importante aún, el hecho de que este proceso de mutación semeja al de un artista en su técnica de elaboración plástica. Las referencias míticas de estos dos episodios recuerdan la actividad manual de los escultores, y la importancia sensorial del tacto y la visión: elementos ambos que engañan al artesano y espectador. En esta vía, el mito de Pigmalión es, de entre todos los mitos que habitan en la Metamorfosis, la piedra angular de todo este caleidoscopio mineral: "Pygmalion is the most conspicuous nexus between (I) the stone imagery on the one hand and (2) the love motif on the other: I. The story employs the essential elements of the vocabulary forecast by the Deucalion episode" (Bauer I2). ${ }^{\mathrm{I}}$

El mito de Deucalión y Pirra emplea el calificativo de "fabricator" para designar en primer lugar la habilidad artística de la divinidad artífice del mundo. Luego, Deucalión y Pirra adquieren por la línea paterna de su 
progenitor Prometeo esas mismas destrezas artesanales, "paternis artibus". Realizan un acto semejante al de un escultor y la creación de una estatua con la transformación de las piedras en humanos al final de la historia: "forma potest hominis, sed, uti de marmore coepta, / non exacta satis rudibusque simillima signis" ("podía ya parecer aquello un algo de figura humana, aunque todavía no resultaba evidente, sino que era como una obra empezada en mármol, no bien terminada aún y semejante a las estatuas a medio hacer"; I.405-406; Ruiz 23). Igualmente, este ambiente artesanal de modelación y de cambio de la materia que se respira en el mito de Deucalión y Pirra reaparece en la historia mítica de Níobe al describirse su transformación en piedra como si fuera una estatua de mármol: "liquitur, et lacrimis etiam nunc marmora manant" ("se licúa, y todavía ahora siguen los mármoles manando lágrimas"; VI.3I2; Ruiz 30). Estos dos mitos que ejemplifican los dos caminos posibles de un cambio: de inanimado a animado, y viceversa, resaltan la valía de un artesano que participa en estas transformaciones de la materia.

La Metamorfosis no posee historia alguna que cuente el paso de un personaje a un estado de vidrio. La inclusión de estos dos mitos, que tienen como base común el elemento de la piedra, conciencia al lector sobre los cambios que se pueden experimentar: a partir de la materia rocosa, y en esta misma. Ambas narraciones conectan con la mudanza imaginaria de Tomás Rodaja en un personaje constituido de vidrio, sin olvidar que el elemento de la piedra es el tropo maestro de la Metamorfosis. La sustancia del vidrio de la $N L V$ se contrapone a la piedra en lo que respecta a su naturaleza translúcida y maleable, pero importa más desde un punto de vista literario. Dentro de la $N L V$ es la materia por excelencia de la mutación del personaje principal, y con ello, se opone a la valía letrada que la piedra tiene en la Metamorfosis. Es decir, el símbolo del vidrio aparece como cimiento de la $N L V$, y se le enfrenta a la piedra por su importancia metafórica en la Metamorfosis. A su vez, este material inorgánico tiene la particularidad de que semeja un parecido fónico con la onomástica del poeta Ovidio: vidrioOvidio. En esta línea, la $N L V$ introduce a un personaje que imagina su materia corpórea hecha a partir de un elemento que encierra en su nomenclatura ecos ovidianos.

Tomás Rodaja adquiere, cuando se imagina todo compuesto de vidrio, un nuevo nombre que recoge toda su corporalidad cristalina, licenciado Vidriera. A partir de aquí, el empleo repetido de este renombre provoca que se olvide la materia de vidrio de su ser, la cual se relega a su onomástica. No obstante, a pesar de la creación de este apelativo no hay que ignorar la materia del vidrio, de donde surge este vocablo, "vidriera", y cuya creación le rinde tributo. Ahora, "vidrio" se altera en: "vidriera", que responde a los 
parámetros onomásticos de creación lingüística dentro del universo literario de Cervantes.19 La $N L V$ encierra un contexto cultural en el que predominan subtextos ovidianos con temática transformacional para que Cervantes introduzca su transfiguración literaria, la de Tomás Rodaja en un pseudo-Ovidio. Tomás Rodaja se convierte en un camuflado ente ovidiano que encierra un parecido con la onomástica del poeta desde un punto de vista léxico, y mediante esta inusual naturaleza vidriosa. El crítico Julio Baena propone la tríada de "alto, sonoro, y significativo" como la clave para descifrar el enigma onomástico que se oculta en los nombres cervantinos (51); calificativos que se aprecian en el nombre del licenciado Vidriera. Igualmente, propone un esquema sucinto para agrupar algunos de los procedimientos lingüísticos que Cervantes emplea en la confección de sus nombres: "a. manipular el sonido de las palabras. -caso r: similitud fonética. -global (Benengeli-berenjena; Aldonza-Dulcinea). -por rima (don FernandoPandafilando; Clodio-odio). -caso 2: unión de dos palabras castellanas (Rocín-ante)" (Baena 5I-52).

La ligera taxonomía que el crítico esquematiza aclara sus posibles juegos léxicos y sonoros. Ayuda a vislumbrar el gran número de combinaciones sintácticas, semánticas y fonéticas que residen en la formación de la onomástica del mundo cervantino. También, Cervantes emplea nombres de personajes literarios que pertenecen al acervo caballeresco o la cultura grecorromana. Se inventa esos mismos nombres para parodiarlos, de manera que: "Todos estos apelativos demuestran la relación filial que ha tenido Cervantes con la literatura, destacándose de manera especial, tanto la literatura caballeresca real así como la paródica, que inventa don Quijote gracias a su entusiasta imaginación" (Acevedo I87). ${ }^{20}$ En este caso, la eliminación de la primera "o" en el nombre de Ovidio deriva en vidio, y la añadidura de una " " crea el vocablo vidrio, por lo que se crea este juego de paronomasia en la $N L V$ : Ovidio a Vidio, después Vidrio, y, finalmente, vidrio. En este sentido, Cervantes adquiere las mismas habilidades artesanas que se refieren en los dos mitos anteriores. Imita, moldea y adapta con agudeza al otro genio de la literatura transformacional, Ovidio, en la naturaleza vítrea del licenciado Vidriera. Como resultado, se obtiene a un individuo que goza de una corporeidad que posibilita una exégesis dentro del cosmos alegórico de la Metamorfosis.

En conclusión, los mitos ovidianos de Deucalión y Pirra, y Níobe permiten reconsiderar la transformación psíquica de Tomás Rodaja en vidrio. Estos se entretejen en dos escenas después de que Tomás Rodaja se imagina todo de vidrio: cuando unos niños le arrojan piedras, y su encuentro con una ropera salmantina. Así pues, la colocación inicial de estos mitos sirve para teorizar y reflexionar sobre el proceso de mutación como labor 
artística. Ovidio y Cervantes despliegan sus habilidades literarias para ofrecer sus respectivas transformaciones, y metamorfosear así a sus personajes respectivamente. La sustancia vítrea de la corporeidad de Tomás Rodaja en la $N L V$ invita a cuestionar la razón de su elección, y contrasta con el tropo maestro de la Metamorfosis, la piedra. En este sentido, la similitud fonética del vocablo vidrio con el nombre del poeta Ovidio sugiere, dentro del universo creacional cervantino de nombres, una semejanza que propone la reencarnación de un pseudo-Ovidio en la persona vitrificada de Vidriera. La $N L V$ despliega en esta sección de su historia los pilares teóricos sobre el proceso artístico de la metamorfosis literaria, y qué mejor manera de hacerlo que con la mutación del creador de las transformaciones letradas: Publio Ovidio Nasón. Así pues, este trabajo ofrece un análisis que encuadra la locura y cambio del personaje del licenciado Vidriera, que se imagina todo de vidrio, en línea con dos mitos de la Metamorfosis de Ovidio. Ello muestra la concienzuda elaboración de esta mutación y su matriz ovidiana que reiteran el profundo interés literario de Cervantes por la obra de Ovidio. Este estudio valora la importancia de seguir rastreando la influencia ovidiana en Cervantes como modelo a imitar, modelar, y reinventar. Con ello, se demuestra el diálogo creativo que Cervantes establece con Ovidio, y que propone estudiar la $N L V$ como un taller de experimentación y creación literaria. Cervantes con la elección de esta sustancia vítrea demuestra su ingenio y finura letrada a la hora de enfrascar en ella una lectura ingeniosa del nombre del poeta por excelencia de las mutaciones. Este se eleva con esta transformación y juego fonético como el padre de las metamorfosis literarias al engendrar a un avatar de psique ovidiana en uno de sus personajes de ficción.

Georgia Southern University

NOTAS

I Véanse por ejemplo: Tristia 3.3-59-64; y Metamorfosis XV.872-88o. El poeta reflexiona en ambos lugares sobre la posteridad de su obra y persona. La sección de Pitágoras en el libro XV encierra toda su filosofía acerca de la mutabilidad y durabilidad del ser, y también su obra poética.

2 Colin Burrow y Raphael Lyne trazan el influjo de Publio Ovidio Nasón en las letras anglosajonas y francesas. Por su parte, Rudolph Schevill aborda la literatura hispánica, y es a día de hoy la obra más completa.

3 Las iniciales $N L V$ se usan de aquí en adelante para abreviar el título de la novela, Novela del licenciado Vidriera. 
4 Empleo a lo largo de todo el trabajo el artículo "la" para referirme a la obra, Metamorfosis de Ovidio, y no al conjunto de mutaciones que esta recoge.

5 'Pseudo puede equivaler a 'falso', 'que pretende hacerse pasar por', 'que imita a', 'erróneamente atribuido a', etc..." (Martín 22r).

6 Miguel de Cervantes se convierte asimismo en el poeta Publio Ovidio Nasón en uno de los diez sonetos que preceden al capítulo primero de la primera parte de Don Quijote de la Mancha: "Salve otra vez, ¡oh Sancho!, tan buen hombre, / que a solo tú nuestro español Ovidio, / con buzcorona te hace reverencia" (Cervantes 9I). Interesa notar que Cervantes compara las transformaciones que padecen los personajes principales de la Novela de La ilustre fregona con las de Ovidio en la Metamorfosis, y estima las suyas como superiores: "He aquí tenemos ya - en buena hora se cuente a Avendaño hecho mozo del mesón, con nombre de Tomás Pedro, que así dijo que se llamaba, y a Carriazo, con el de Lope Asturiano, hecho aguador: transformaciones dignas de anteponerse a las del narigudo poeta" (159). Estos ejemplos entrevén la aspiración literaria de Cervantes a sobrepasar al poeta latino. En esta línea, John C. Parrack señala que Sánchez de Viana se superpone a Ovidio en los sonetos que anteceden a su traducción Anotaciones: “Collectively, they transform Sánchez de Viana into a classical figure that explains Ovid, gives new life to Ovid, makes Ovid relevant, and triumphs over Ovid" (25).

7 Los comienzos de la crítica cervantina de la Novela del licenciado Vidriera y por orden cronológico argumentan un interés de Cervantes por la psicología (Gutiérrez-Noriega), el síndrome de la locura (Singer), las teorías aristotélicas de la melancolía (Green; Casa), y ponzoñas (Messik). A su vez, Dunn explora la curiosidad y dedicación de Tomás Rodaja al estudio, mientras que El Saffar analiza el conflicto de este personaje con su ambiente. La filosofía cínica del Renacimiento y la Philosophia y Respublica Christiana de Erasmo emergen a partir de su caída moral en la búsqueda del conocimiento (Forcione 225-316). La novela refleja los planteamientos básicos del género picaresco (Gerli), y se vislumbra el incesante batallar del personaje principal con su origen converso (Ricapito). La clase social de Tomás Rodaja y su identidad sirven como vehículos para criticar la política imperial en declive del imperio español (Clamurro). Se indaga así la repercusión de la sátira literaria (Rupp), y con ello la novela encierra en sí misma un pasatiempo de afinación mental, habilidad lingüística e ingenio (Scham 2I9). También se aborda el perspectivismo y la amistad (Puig), el empleo del emblema renacentista como experimento verbal (Aylward I88-205) y la doctrina paulina del libre albedrío (Blasco). Para un enfoque de la estructura artística y 
meditada de la novela, interesa consultar los trabajos de Joaquín Casalduero, Armand E. Singer ("Cervantes' Licenciado Vidriera"), Frank P. Casa ("The Structural Unity"), Avalle-Arce , Gwynne Edwards y Edward H. Friedman.

8 Este influjo ovidiano sigue todavía en vigor gracias a los esfuerzos académicos y las numerosas publicaciones del cervantista Frederick de Armas. A este respecto hay que mencionar su edición del volumen Ovid in the Age of Cervantes, que demuestra con sus quince contribuciones la gran valía, recepción y estudio de la producción literaria de Ovidio en el Siglo de Oro español: “Ovid's tales, his powerful images, and startling transformations were imitated and refashioned by the leading Spanish writers of the period: Cervantes, Calderón de la Barca, Góngora, Lope de Vega, and Quevedo" (De Armas IX). Consúltense también los trabajos de Vicente Cristóbal para la aetas Ovidiana en la España medieval a partir del siglo XIII; y Barry Taylor para la alegoresis ovidiana en el Siglo de Oro. La nota primera de mi trabajo "El engaño del sentido ocular en la Novela del celoso extremeño: Pigmalión y Narciso" complementa también la influencia de Ovidio en Cervantes según la crítica literaria, y explora el influjo de dos mitos ovidianos en dicha novela.

9 Harry Sieber indica las fuentes ovidianas de las respuestas posteriores del licenciado Vidriera a las preguntas de varios estudiantes con respecto a los poetas y la poesía en sus notas 54, 55 y 56. Lo llamativo de este diálogo entre el licenciado Vidriera y varios estudiantes es que el tema principal de la charla, el ser poeta y la poesía, va en línea con la transformación de este personaje, Tomás Rodaja, en un pseudo-Ovidio.

IO El texto enfatiza la importancia de la materia del vidrio con la presencia de este vocablo por ocho veces hasta que el personaje adquiere el nombre de licenciado Vidriera. Daniel Heiple sigue los pasos de Rodríguez Marín y Otis Green en su estudio sobre la influencia que los tratados médicos de la época ejercieron sobre la pluma de Cervantes cuando creó el personaje de Don Quijote. Sin embargo, señala en lo tocante a la $N L V$ : “Cervantes other famous portrayal of a madman, that of Tomás Rodaja in El licenciado Vidriera, does not use this kind of scientific background. It is true that he mentions the diet and eating habits of the mad licenciado, but they are not introduced for their humoral characteristics, but to underline the strangeness of the heroe's insanity" (70).

II La historia de este mito fue muy conocida en la antigüedad. Las versiones de Apolodoro (I.7.2) e Higinio (152) ofrecen detalles curiosos sobre el mito (Anderson I8I).

I2 Hay que mencionar que las piedras ocupan el segundo lugar de frecuencia en la Metamorfosis, y luego, los pájaros (Lafaye 247-48). 
I3 Los números romanos apuntan al libro de la Metamorfosis de Ovidio, y los arábigos a sus versos correspondientes. Por su parte, la numeración de Ruiz señala las páginas de la traducción.

I4 El verbo en latín es fabrǐcō, de donde derivan las palabras fraguar, forjar, forja, forjador. El sustantivo făbrǐca significa: "f., oficio, arte: aeraria, materiaria f., arte de trabajar el bronce, la madera || [en partic.] la Arquitectura || trabajo de artesanía, artístico [acción de fabricar y objeto fabricado]; obra de arte || [fig.] invención, maquinación, treta, astucia || fábrica, taller || [en especial] fragua, forja: Vulcani fabricae, las fraguas de Vulcano || construcción, edificio" (Segura 267). Y a su vez, el sustantivo en masculino, făběr,-brī: "m., obrero, artesano, artífice [refer. gralte. al que trabaja con materiales duros: metales, piedras, madera, marfil, etc.]: praefectus fabrum [gen. pl.], el capataz de los obreros || [el sentido va precisado generalmente por un epíteto]: f. ferrarius, herrero; f. aerarius, calderero, latonero; f. tignarius, carpintero." (267). También, la divinidad, justo en el momento que se narra el origen del hombre, emplea el calificativo de: "ille opifex rerum" ("el artífice de la naturaleza"; I.79; Ruiz 9). La etimología y significado del término: "opifex", apunta a ese cosmos artesanal: "ŏp̌̌fex, ǐcis [opus, facı̄ō], $m$. $f$., el que hace una obra; creador, autor, artífice; ille opifex rerum, el autor de todas las cosas; o. mundi, Deus, Dios, el creador del mundo || trabajador, obrero; artesano, artista" (Segura 490).

I5 "[Q]uam satus Iapeto mixtam pluuialibus undis / finxit in effigiem moderantum cuncta deorum" ("esa tierra que el vástago de lápeto modeló, mezclándola con aguas de lluvia, hasta darle la figura de los dioses que todo lo gobiernan"; I.82-83; Ruiz Io). El empleo del verbo "finxit" le confiere esa habilidad artística: "fingō, -ere, finxī, fictum, tr., modelar [en arcilla]; dar forma, formar; moldear; hacer, construir, componer: ceram f., modelar la cera; favos f., hacer panales [de miel]; $e$ cera f., modelar con cera || esculpir: Herculem f., modelar, esculpir un Hércules; ars fingendi, el arte de modelar, la escultura" (Segura 283). "PROMETEO: Hábil artesano, Prometeo es considerado el creador de los primeros hombres, a los que modeló con barro. Sin embargo, es mucho más conocido como benefactor de la humanidad, a la que socorrió en dos ocasiones desafiando la cólera de Zeus" (Martin 369).

I6 Las dos palabras que refuerzan la habilidad artesanal de Prometeo son "artibus y formatae". Véase el Diccionario etimológico latino-español (57; 290-91).

I7 'As the stones take shape, they become 'natura ... mitior,' and when Ovid says, 'mollirique mora mollitaque ducere formam' he is not only greeting us with a barrage of softening 'm's, but he is also using one of his favorite 
puns, since forma means both 'form' and 'beauty.' Human life here defines itself as beautiful in that it is soft, flexible, and formed" (Barkan 643).

I8 El propio Ovidio se valora a sí mismo metafóricamente como el escultor capaz de ablandar las piedras en Fastos III.829-34: "No vosotros, maestros, un grupo casi privado de censo, la despreciéis: ella atrae nuevos discípulos; y tú, que le das al cincel y pintas cuadros con colores al incausto, y tú, que con hábil mano das formas suaves a las piedras. Es diosa de mil ocupaciones. Desde luego, es la diosa del poema; si me lo merezco, que asista a mis afanes amigablemente" (Segura I27). El episodio que precede a la historia de Pigmalión sirve para introducir al poeta en la figura del escultor: "Furthermore, the figurative equation between the sculpture of Pygmalion and the art of the poet may be intentionally foreshadowed in our text by the appearance of the curious Propoetides, already a part of both the stone image and the love motif, who are usually explained to be the daughters of an unknown Propoetus, but who are actually placed pro poeta in the series of episodes in order to alert the attentive reader to the allegory before him" (Bauer 13-I4).

I9 Véase el pionero trabajo de Leo Spitzer que explora la ambivalencia, o como dice el crítico: polionomasia, de los nombres de varios personajes de Don Quijote. Interesan las diferentes disputas lingüísticas y etimológicas que ocurren en la novela, y que revelan en su conjunto la conciencia de perspectivismo lingüístico de su autor.

20 La bibliografía que trata sobre los mecanismos onomásticos en las obras de Cervantes, y Don Quijote, es abrumadora. Al respecto hay que mencionar que: "No obstante, en el caso de Cervantes, el uso hermenéutico analítico de la onomástica es mucho más defensible y hasta está indicado. Es evidente que el autor del Quijote gozaba mucho de la creación de nombres de su propia cosecha, por regla general altisonantes, melifluos, y convincentes" (Sullivan 74). El primer episodio de Don Quijote cuando nombra a su montura, personaje y dama, sirve para ejemplificar la importancia de este proceso, y demuestra el ingenio agudo de Cervantes en la creación de nombres. También, interesa el episodio XVIII de esta primera parte donde el personaje confunde la manada de corderos con ejércitos, y se inventa el nombre de un buen número de caballeros: "Y has de saber, Sancho, que este que viene por nuestra frente le conduce y guía el gran emperador Alifanfarón, señor de la grande isla Trapobana; este otro que a mis espaldas marcha, es el de su enemigo, el rey de los garamantas, Pentapolín del Arremangado Brazo, porque siempre entra en las batallas con el brazo derecho desnudo" (Cervantes, Don Quijote I, 228). Un claro ejemplo de una lectura artificiosa de un nombre corre a cargo de Sancho Panza cuando confunde el nombre del cronista arábigo, Cide 
Hamete Benengeli por Cide Hamete Berenjena (Cervantes, Don Quijote II $2,44)$.

\section{OBRAS CITADAS}

Anderson, William S., ED. Ovid's Metamorphoses. Books I-5. Norman: U of Oklahoma $\mathrm{P}, \mathbf{1 9 9 7 .}$

AVALle-ARCE, J.B. And E.C. Riley, E Ds. Suma Cervantina. London: Tamesis, 1973. A y l W R D, E. T. The Crucible Concept. Thematic and Narrative Patterns in

Cervantes's Novelas ejemplares. Cranbury: Associated University Presses, I999. BAEnA, Julio. "Modos del hacedor de nombres cervantino: El significado de 'Cide Hamete Benengeli.'” Indiana Journal of Hispanic Literatures 2.2 (I994): 48-6I.

B ARKA N, LE ONARD. “'Living Sculptures': Ovid, Michelangelo and the Winter's Tale." ELH 48.4 (I98I): 639-67.

BA U E R, D OU G LAS F. "The Function of Pygmalion in the Metamorphoses of Ovid." Transactions and Proceedings of the American Philological Association 93 (I962): I-2I.

B L A SC 0, J AVI E R. “El licenciado Vidriera: la inestabilidad onomástica y la polémica 'de auxiliis.'” Cincinnati Romance Review 37 (2014): 6-23.

BU R R ow, C O L In. "Re-embodying Ovid: Renaissance Afterlives." The Cambridge Companion to Ovid. Cambridge: Cambridge UP, 2002.30I-I9.

CAS A, FRAn K P. The Dramatic Craftsmanship of Moreto. Cambridge: Harvard UP, I966. 30-52.

-. "The Structural Unity of El licenciado Vidriera."Bulletin of Hispanic Studies 4I (I964): 242-46.

Ca A A d u Ro, Jo Aquín. Sentido y forma de las Novelas ejemplares. Buenos Aires:

Coni, 1943 .

Ce RVA n tes, M I G U L DE. El ingenioso hidalgo Don Quijote de la Mancha. Ed. John Jay

Allen. 2 Vols. Madrid: Cátedra, 2004.

—. Novelas ejemplares II. Ed. Harry Sieber. Madrid: Cátedra, 2003.

Clamur ro, william h. Beneath the Fiction. The Contrary Worlds of Cervantes's

Novelas ejemplares. New York: Peter Lang, 1997.

CORDERO DE CiRia, EnRiQue. "Iconografía de Faetón en España." Goya: Revista de arte $\mathrm{I} 85$ (I985): $274-8 \mathrm{I}$.

CR ISt ó B Al, vicente. "Ovid in Medieval Spain." Ovid in the Middle Ages. Eds. James G. Clark, et al. Cambridge: Cambridge UP, 20II. 23I-56.

De armas, FREDERICK A., E D. Ovid in the Age of Cervantes. Toronto: U of Toronto P, 2010.

D Un N, PE T E R N. “Las Novelas ejemplares." Avalle-Arce y Riley, 8I-II8. 
E DWA RDS, GW YN E. “Cervantes's El licenciado Vidriera: Meaning and Structure.”

Modern Language Review 68 (1973): 559-68.

E L SAFFAR, RUTH S. Novel to Romance: A Study of Cervantes's Novelas ejemplares.

Baltimore: The Johns Hopkins UP, I974.

ENCINAR, MARÍA ÁNGELES. "La formación de personajes en tres novelas ejemplares: El licenciado Vidriera, El celoso extremeño, y La fuerza de la sangre." Cervantes 15 (1995): 70-8I.

Forcione, ALBAn K. Cervantes and the Humanist Vision: A Study of Four Exemplary Novels. Princeton: Princeton UP, 1982.

FRIED MAN, EDWARD H. “Conceptual Proportion in Cervantes' El licenciado Vidriera." South Atlantic Bulletin 34 (I974): 5I-59.

GARCÉs, maría Antonia. "Delirio y obscenidad en Cervantes: el caso Vidriera." Actas del XII Congreso de la Asociación Internacional de Hispanistas. Birmingham 1995. Eds. Aengus M. Ward, et al. Birmingham: Department of Hispanic Studies, 1998.

G A R CíA L O R CA, F RA N IS C o. "El licenciado Vidriera y sus nombres." Revista Hispánica Moderna 3г (1965): 159-68.

Ge RLi, mich a L E. Refiguring Authority: Reading, Writing, and Rewriting in Cervantes. Lexington: UP of Kentucky, 1995. I0-23.

gonzále z vázquez, jo sé, trad. Tristes y Pónticas. Por Publio Ovidio Nasón. Madrid: Gredos, 1992.

gONZÁlez de Amezúa y mayo, agustín. Cervantes creador de la novela corta española. 2 vols. Madrid: CSIC, 1958.

Gó RRIZ, MARIA n o. "Cervantes, psicólogo y psiquiatra." Revista de la Universidad (Panamá) 27 (I947): 8I-IO2.

GREE N, о TIS H. "El licenciado Vidriera: its relation to the Viaje del Parnaso and the Examen de ingenios of Huarte." Linguistic and Literary Studies in Honor of Helmut A. Hatzfeld. Ed. Alessandro S. Crisafulli. Washington: Catholic U of America P, 1964. 213-20.

ha insworth, G. "La source du Licenciado Vidriera." Bulletin Hispanique 32.I (1930): 7O-72.

He IP LE, DANiEl L. "Renaissance Medical Psychology in Don Quijote." Ideologies and Literature 2.9 (I979): 65-72.

H IDALG o, JoSÉ MANUE L. "El engaño del sentido ocular en la Novela del celoso extremeño: Pigmalión y Narciso." Revista de Estudios Hispánicos 46 (2012): 50525.

—. "El ethos elegíaco de Tomás Rodaja en la Novela del licenciado Vidriera." Cervantes 38.2 (2018): IOI-27.

нu т , sylvia. "The Medusa Interpolation in the Romance of the Rose:

Mythographic Program and Ovidian Intertext." Speculum 62.4 (1987): 865-77. 
JAn AN, miCAE La. "'There Beneath the Roman Ruin Where the Purple Flowers Grow': Ovid's Minyeides and the Feminine Imagination." The American Journal of Philology II5.3 (1994): 427-48.

J OSE T, J ACQUES. "Bipolarizaciones textuales y estructura especular en El licenciado Vidriera." Cervantes, su obra y su mundo: Actas del I Congreso Internacional sobre Cervantes. Ed. Manuel Criado del Val. Madrid: EDI-6, I98I. 357-64.

-. "Libertad y enajenación en El licenciado Vidriera." Actas del VII Congreso de la Asociación Internacional de Hispanistas. Venecia 1980. Ed. Giuseppe Bellini. Roma: Bulzoni Editore, 1982. 613-19.

L AFAY E, GE O R G S. Les métamorphoses d'Ovide et leurs modèles grecs. 1904. Hildesheim: Olms, I97I.

Lyne, RA Pha el. "Love and Exile after Ovid." The Cambridge Companion to Ovid. Ed. Philip Hardie. Cambridge: Cambridge UP, 2002. 288-300.

LUGO ACEVED 0, MARÍA LUiSA. “'Yo sé quién soy': Acerca de cómo Don Quijote construye su identidad a través del nombre." Cervantes 33.2 (2013): 173-92.

M A R I N, RENÉ. Diccionario de la mitología griega y romana. Madrid: Espasa Calpe, 1996.

MART Ín PUENTE, CRISTINA. "Tratamiento de las obras anónimas y de dudosa autoría en la filología latina." Mundus vult decipi: Estudios interdisciplinares sobre falsificación textual y literaria. Ed. Javier Martínez. Madrid: Ediciones Clásicas, 20I2. 213-26.

messick, A lan. “Tomás Rodaja: A Clinical Case?” Romance Notes II (I970): 623-28. NEW Lands, CAR OLE. "The Simile of the Fractured Pipe in Ovid's Metamorphoses 4." Ramus I5.2 (1986): 143-53.

ovidio nasón, publio. Metamorfosis. 3 Vols. Trad. Antonio Ruiz de Elvira. Madrid: CSIC, 1990.

PARRACK, Jo H N C. "Mythography and the Artifice of Annotation: Sánchez de Viana's Metamorphoses (and Ovid)." Ed. Frederick A. de Armas. 20-36.

PU IG, I D Y A. "Relaciones humanas en Cervantes: El licenciado Vidriera y El celoso extremeño: las excepciones que confirman la regla." RILCE: Revista de Filología Hispánica I4.I (1998): 73-88.

RIC API T 0, Jo SE PH v. Cervantes's Novelas ejemplares. Between History and Creativity. West Lafayette, Indiana: Purdue UP, 1996.

RILEY, E.C. "Cervantes and the Cynics (El licenciado Vidriera and El coloquio de los perros)." Bulletin of Hispanic Studies 53 (1976): 189-99.

R UP P, s T E P E n. "Soldiers and Satire in El licenciado Vidriera." A Companion to Cervantes's Novelas ejemplares. Ed. Stephen Boyd. Woodbridge: Tamesis, 2005. 134-47.

Salzman-mit chell, Patricia. “A Whole Out of Pieces: Pygmalion's Ivory Statue in Ovid's Metamorphoses." Arethusa 4I.2 (2008): 29I-3II.

Sant a BIBLIA. Ed. Dr. Evaristo Martín Nieto. Madrid: San Pablo, I989. 
SCHAM, M ICHAE L. Lector Ludens: The Representation of Games and Play in Cervantes. Toronto: $\mathrm{U}$ of Toronto P, $20 \mathrm{I} 4$.

SCHEV IL L, RU D O PH. Ovid and the Renascence in Spain. Berkeley: U of California P, I9I3.

Segura ramos, Bartolomé, trad. Fastos. Por Publio Ovidio Nasón. Madrid: Gredos, I988.

SE G RE, CE SARE. "La estructura psicológica de El licenciado Vidriera." Actas del I Coloquio Internacional de la Asociación de Cervantistas. Alcalá de Henares 1988. Barcelona: Anthropos, 1990. 53-62.

SingER, A R M A D E. "Cervantes' Licenciado Vidriera: Its Form and Substance". West Virginia University Philological Papers 8 (195I): 13-3I.

—. "The Sources, Meaning, and Use of the Madness Theme in Cervantes' El licenciado Vidriera." West Virginia University Philological Papers 6 (1949): 31-53. S P IT ZE R, LE o. "Perspectivismo lingüístico en el Quijote." Lingüística e historia literaria. Madrid: Gredos, I968. I35-87.

SULLIVAN, HENRY WELLS. "Altisidora: ¿Cómo 'regalo del más alto' acelera la cura de don Quijote?" La mujer y su representación en las literaturas hispánicas. Actas Irvine 92. Ed. Juan Villegas Morales. 5 vols. Irvine: Asociación Internacional de Hispanistas, University of California, I994. Vol. 2. 74-8I.

TA Y LOR, B ARRY. "Lecturas alegóricas de las Metamorfosis de Ovidio en la España del Siglo de Oro." Las metamorfosis de la alegoría. Discurso y sociedad en la Península Ibérica desde la Edad Media hasta la Edad Contemporánea. Eds. Rebeca Sanmartín y Rosa Vidal. Madrid: Iberoamericana Editorial Vervuert, 2005. $225-48$.

VALLEJ O NÁJERA, A. Literatura y psiquiatría. Barcelona: Editorial Barna, I95O. WISE, VALERIE M. "Flight Myths in Ovid's Metamorphoses: An Interpretation of Phaeton and Daedalus." Ramus 6.I (1977): 44-59. 